\title{
Research Paper: Influence of Fear of Falling Severity Measured by Falls Efficacy Scale-International on Gait and Balance Performance After Chronic Stroke
}

\author{
Mania Sheikh $^{1}$ (D), Hossein Asghar Hosseini ${ }^{1^{*}}$ (D) \\ 1. Department of Physical Therapy, School of Paramedical Sciences, Mashhad University of Medical Sciences, Mashhad, Iran.
}

Citation: Sheikh M, Asghar Hosseini H. Influence of Fear of Falling Severity Measured by Falls Efficacy Scale-International on Gait and Balance Performance After Chronic Stroke. Iranian Rehabilitation Journal. 2020; 18(1):73-80. http://dx.doi. org/10.32598/irj.18.1.911.1

http://dx.doi.org/10.32598/irj.18.1.911.1

Article info:

Received: 25 May 2019

Accepted: 23 Sep 2019

Available Online: 01 Mar 2020

Keywords:

Stroke, Gait, Balance, Fear, Accidental falls

\section{ABSTRACT}

Objectives: Fear of falling (FoF) is a common complication after stroke affecting gait and balance performance in individuals with a history of stroke. The current study aimed at determining the influence of the level of FoF on gait and balance performance after chronic stroke.

Methods: A total of 76 persons with chronic stroke (including 41 women), who had no cognitive impairment and could walk at least 10 meters without walking aids, participated in this cross-sectional observational study. The participants walked at their preferred speed to calculate gait asymmetry ratios for stance time, swing time, and step length. The Asymmetry Index determined standing balance asymmetry measures of medial-lateral and AnteriorPosterior (AP) Center of Pressure (CoP) velocity. The Falls Efficacy Scale-International (FES-I) evaluated the severity of FoF. Thirty-one participants had no FoF, 25 had low FoF, and 20 had high FoF. A multivariate analysis of variance test was used to compare standing balance and gait parameters among 3 groups. The Pearson correlation test was also used to define the relationships between FES-I and gait and standing balance asymmetry measures in individuals with FoF.

Results: The Mean \pm SD age of the participants was $57.53 \pm 9.28$ years. The Mean \pm SD time since stroke onset was $17.29 \pm 3.6$ months. The participants with a high FoF had a significantly more asymmetrical swing time and step length and higher AP CoP asymmetry compared to those without $\mathrm{FoF}(\mathrm{P}=0.02, \mathrm{P}=0.007, \mathrm{P}=0.03$, respectively). Increased FES-I was related to increased swing time $(\mathrm{r}=0.65, \mathrm{P}=0.001)$, step asymmetry $(\mathrm{r}=0.45, \mathrm{P}=0.002)$, and AP CoP asymmetry ( $\mathrm{r}=0.44, \mathrm{P}=0.003)$.

Discussion: A high level of FoF affects gait and balance asymmetry after chronic stroke. Future research to develop therapeutic programs should consider the effects of FoF.

* Corresponding Author:

Hossein Asghar Hosseini, PhD.

Address: Department of Physical Therapy, School of Paramedical Sciences, Mashhad University of Medical Sciences, Mashhad, Iran.

Tel: +98 (935) 2218407

E-mail:hosseiniha@mums.ac.ir 


\section{Highlights}

- Chronic stroke survivors with high FoF have greater gait and standing balance asymmetry than those without FoF.

\section{Plain Language Summary}

High FoF is related to gait and balance disturbance in individuals with chronic stroke. Health care personnel, who see persons with stroke, can address FoF within therapeutic programs to help patients resume a more functional gait and balance.

\section{Introduction}

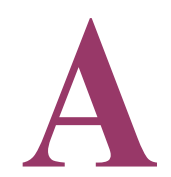

$\mathrm{n}$ injury resulting from a fall is a potential medical complication at all stages after stroke $[1,2]$. In addition to physical injuries, falls may be associated with psychological consequences known as a fear of falling (FoF) [3]. FoF can seriously limit activities and mobility by diminishing the sense of well-being and reducing social interactions [4]. Previous literature indicated that FoF could be helpful as a disability predictor among older adults [5].

The FoF influences gait and balance in elderly people even in the absence of fall history [6]. Chamberlin et al. reported that decreased stride length, decreased gait velocity, and increased time of double support phase were related to FoF in older adults and, thus, reflect stabilizing adaptations related to $\mathrm{FoF}$ [4]. Allali et al. indicated that FoF was a specific fall predictor in older adults with postural instability and gait difficulty [7]. A limited number of studies have investigated the relationship between FoF and various features of gait and balance after stroke. A recent study by Schinkel-Ivy et al. found no significant difference in gait and balance measures between fear and no fear of falling groups [8]. The lack of findings in their study may be related to the FoF evaluation method [9]. Therefore, in the current study, we decided to determine the level of FoF, using Falls Efficacy Scale-International (FES-I), which evaluates the FoF severity in various physical and social activities [10].

Since many physical impairments may contribute to increased balance and gait asymmetry after stroke [11-13], we assumed that these impairments, sensed by individuals with asymmetry, may lead to FoF. On the other hand, individuals with a high FoF may increase the muscular activity of the non-affected leg or reduce the time spent standing on the affected leg in response to this fear, and these personal adjustments may lead to imbalance and gait asymmetry. Therefore, the hypothesis of this study states that individuals without FoF compared to those with different levels of FoF would represent greater asymmetry for gait and standing balance measures. Furthermore, increased FoF levels were expected to be related to the increased asymmetry of gait and balance measures.

The current study aimed at comparing the gait asymmetry and standing balance measures between individuals with stroke relative to their level of FoF. This study also determined the relationships between the level of FoF and asymmetry of gait and standing balance measures in participants with FoF.

\section{Methods}

\section{Participants}

In this observational cross-sectional study, based on convenience sampling, 76 persons with chronic stroke were recruited from the rehabilitation clinic at the Quaem Hospital, Mashhad, Iran, between June 2011 and December 2013. Volunteers were screened by a physician based on the inclusion and exclusion criteria. Stroke diagnosis was determined according to the World Health Organization (WHO) definition [14]. The inclusion criteria included first stroke experience $>6$ months ago, a Mini-Mental State Examination score above 24 [15], and an ability to walk at least 10 meters without assistive devices. The exclusion criteria included instability in medical conditions, cognitive problems or severe aphasia, and a history of psychiatric illness.

The ankle plantar flexors spasticity of the paretic lower limb was assessed, using the Modified Ashworth Scale. The grades range between 0 and 5, with 0 indicating normal muscle tone and 5 demonstrating a fixed contracture [16]. The Modified Ashworth Scale grades were $\leq 2$ in all participants. The level of motor impairments of the paretic lower limb was assessed with the Fugl-Meyer Assessment [17]. This assessment is valid and reliable 
in stroke survivors [17]. The Fugl-Meyer Assessment scores were more than 18 in all participants.

All participants were divided into 3 groups based on the level of FoF that was measured by the FES-I; participants without FoF $(n=31)$, with a low FoF $(n=25)$, and with a high FoF $(n=20)$. A priori power analysis was used to calculate the required sample size $(\alpha=0.05$; $\beta=0.8$ ). FES-I was used to evaluate FoF when carrying out 16 daily living activities, including various social activities $[10,18]$. The FoF score for each activity was determined, using a 4-point scale. The total range was between 16 and 64. The reliability and validity of FES-I are established in persons with stroke [18]. A score of 16 indicates no FoF. Based on the previous literature, a score of 23 was set as the cut-off point for the distribution of participants to low and high FoF groups [10]. Individuals with scores $>23$ were assigned to the high FoF group, and those with scores $\leq 23$ were assigned to the low FoF group.

\section{Gait and balance assessment}

Standing balance measures were obtained, using two force plates placed side by side ( $<1 \mathrm{~mm}$ of separation). The participants stood with open eyes and their arms at sides in a standardized position (feet were placed at 14 degrees and $17 \mathrm{~cm}$ between the heels) [19] for the duration of the trials (6 trials were recorded and the duration of each trial was $30 \mathrm{~s})$. The distance of each foot from the midline between both plates was the same. For safety, the participants remained in a harness with two straps attached to the ceiling. Force-plate data were sampled at $200 \mathrm{~Hz}$ and, then, filtered via a second-order $10 \mathrm{~Hz}$ low-pass Butterworth filter. Center of pressure $(\mathrm{CoP})$ velocity in both Anterior-Posterior (AP) and medial-lateral (ML) directions were determined for each force-plate. The root means square of CoP velocities in both directions was determined for standing trials. The Asymmetry Index was used to determine the asymmetry of standing balance measures [20].

Asymmetry Index=Non-paretic limb value/ (paretic limb value + non-paretic limb value). This index ranges between 0 and 1 . An index of 0.5 demonstrated perfect symmetry. If the Asymmetry Index is more than 0.5 , the value of that variable is greater for the non-affected leg compared to that of the affected leg [20].

A simple video-based portable tool for gait analysis was used to define the gait asymmetry measures. Previous studies have indicated the validity and reliability of this system [21, 22]. A single conventional camera with a sampling frequency of $60 \mathrm{~Hz}$ was placed perpendicular to the central part, and 4 meters from the walkway with a length of 10 meters. The participants started walking 5 to 6 steps before reaching the central $2 \mathrm{~m}$ of the walkway and 5 to 6 steps after passing the central $2 \mathrm{~m}$ of the walkway to get the constant speed and avoid the effects of acceleration and deceleration. The participants were instructed to perform walking trials at their preferred speed until 12 steps were collected (4-6 walking trials and 2-3 steps in each trial).

Adhesive reflective markers (with a diameter of 25 $\mathrm{mm}$ ) were attached to the heel and toe regions. A software program written in MATLAB R2006a (Natick, MA) determined the values for step length and swing, as well as the stance time of both legs. Heel strike was defined as occurring when the toe marker was within 1 $\mathrm{cm}$ of its height as measured during standing, and toeoff was also defined as occurring when the heel marker exceeded $1 \mathrm{~cm}$ of its height as measured during standing. To define the step length, the 2-dimensional coordinates of heel markers were obtained in the initial foot contact frames in each step and, then, the distance between the two markers was determined. Spatiotemporal asymmetry was determined as the ratio of right and left values with the greater value in the numerator (regardless of the paretic side) [23]. The value of 1.0 demonstrates perfect symmetry. The asymmetry direction was also defined relative to the lower limb, which represented the larger spatiotemporal value [23]. However, the direction of the asymmetry was not statistically analyzed [23].

\section{Statistical analysis}

Demographics and stroke characteristics were described with descriptive statistics. The Shapiro-Wilk test was used to determine the normality of data. The differences in balance and gait parameters among the groups were defined, using a multivariate analysis of variance with Bonferroni correction included with the post hoc test. A 95\% Confidence Interval (CI) was taken for the difference of each balance and gait measure among the three groups. In participants with FoF, the relationships between the level of FoF (FES-I scores) and balance and gait asymmetry measures were determined, using the Pearson correlation coefficients and 95\% CI were calculated for each $r$ value. The SPSS (Chicago, IL) v. 19 was used to perform the statistical analysis. The alpha level was set at $\mathrm{P}<0.05$.

\section{Results}

Table 1 presents the demographics and stroke characteristics of the participants. No significant difference 
Table 1. General characteristics of the study population

\begin{tabular}{ccccc}
\hline \multirow{2}{*}{ Variables } & \multicolumn{4}{c}{ Mean \pm SD/No. } \\
\cline { 2 - 5 } & Without FoF (n=31) & Low FoF (n=25) & High FoF (n=20) & P* \\
\hline Age (y) & $58.19 \pm 10.57$ & $57.38 \pm 9.31$ & $55.9 \pm 7.47$ & 0.69 \\
Sex (male/ female) & $20 / 11$ & $10 / 15$ & $5 / 15$ & 0.65 \\
Weight (kg) & $70.16 \pm 9.89$ & $71.18 \pm 8.42$ & $67.47 \pm 6.37$ & 0.71 \\
\hline Height (cm) & $167.93 \pm 6.42$ & $168.23 \pm 5.79$ & $165.59 \pm 5$ & 0.8 \\
\hline Time since stroke (months) & $17.67 \pm 3.96$ & $18.19 \pm 3.33$ & $16.01 \pm 3.51$ & 0.35 \\
\hline Hemiparetic side, right/left & $17 / 14$ & $13 / 12$ & $11 / 9$ & 0.83 \\
Stroke type (ischemic, hemorrhagic) & $25 / 6$ & $21 / 4$ & $18 / 2$ & 0.77 \\
Modified Ashworth Scale (score/number) & $0.7 ; 1.10 ; 1+0.8 ; 2.6$ & $0.5 ; 1.8 ; 1+0.7 ; 2.5$ & $0.4 ; 1.6 ; 1+0.8,2.2$ & 0.63 \\
\hline Motor impairment & $26.19 \pm 4.09$ & $25.68 \pm 4.3$ & $23.9 \pm 3.37$ & 0.08 \\
\hline
\end{tabular}

Пranian Rehabilitation \ournal

Values are the number for sex, hemiparetic side, stroke type, and also Mean \pm SD for age, weight, time since stroke, height, and motor impairment.

*The difference among the three groups was determined, using multivariate analysis of variance or Kruskal-Wallis test.

was identified in demographic and stroke-related parameters among the three groups. All measures had a normal distribution and all variances were homogeneous. A significant difference was observed in swing time and step length asymmetry ratios between the participants without FoF and participants with a high FoF. Individuals with a high FoF had a significantly higher swing and step asymmetry than the individuals without FoF $(\mathrm{P}<0.021$, CI: -0.112 to $-0.011 ; \mathrm{P}<0.007$, CI: -0.092 to -0.027 , respectively). Individuals with a high FoF also

Table 2. Comparisons of outcomes of participants in three groups

\begin{tabular}{|c|c|c|c|c|c|c|}
\hline \multirow[b]{2}{*}{ Gait and Balance Variables } & \multicolumn{3}{|c|}{ Mean \pm SD/No. } & \multicolumn{3}{|c|}{$\mathbf{P}$} \\
\hline & $\begin{array}{l}\text { No FoF } \\
(n=31)\end{array}$ & $\begin{array}{l}\text { Low FoF } \\
(n=25)\end{array}$ & $\begin{array}{l}\text { High FoF } \\
(n=20)\end{array}$ & No-high & No-low & High-low \\
\hline Swing asymmetry ratio & $1.32 \pm 0.16$ & $1.4 \pm 0.2$ & $1.47 \pm 0.24$ & $0.021^{*}$ & 0.464 & 0.634 \\
\hline $\begin{array}{l}\text { Swing asymmetry direction } \\
\text { (paretic/non-paretic) }\end{array}$ & $31 / 0$ & $25 / 0$ & $20 / 0$ & - & - & - \\
\hline Stance asymmetry ratio & $1.2 \pm 0.1$ & $1.21 \pm 0.11$ & $1.23 \pm 0.13$ & 0.821 & 0.913 & 0.831 \\
\hline $\begin{array}{l}\text { Stance asymmetry directio } \\
\mathrm{n} \text { (paretic/non-paretic) }\end{array}$ & $0 / 31$ & $0 / 25$ & $0 / 20$ & - & - & - \\
\hline Step asymmetry ratio & $1.26 \pm 0.13$ & $1.29 \pm 0.12$ & $1.38 \pm 0.13$ & $0.007^{*}$ & 0.941 & 0.085 \\
\hline $\begin{array}{l}\text { Step asymmetry direction } \\
\text { (paretic/non-paretic) }\end{array}$ & $18 / 11$ & $15 / 10$ & $12 / 8$ & - & - & - \\
\hline AP CoP asymmetry ratio & $0.599 \pm 0.04$ & $0.602 \pm 0.03$ & $0.631 \pm 0.06$ & $0.033^{*}$ & 0.924 & 0.086 \\
\hline ML CoP asymmetry ratio & $0.614 \pm 0.03$ & $0.613 \pm 0.04$ & $0.605 \pm 0.04$ & 0.801 & 0.901 & 0.821 \\
\hline
\end{tabular}

Iranian Rehabilitation Journal

${ }^{*}$ Variables for the participants without FoF $(n=31)$ are significantly different from those with a high FoF $(n=20)$. 


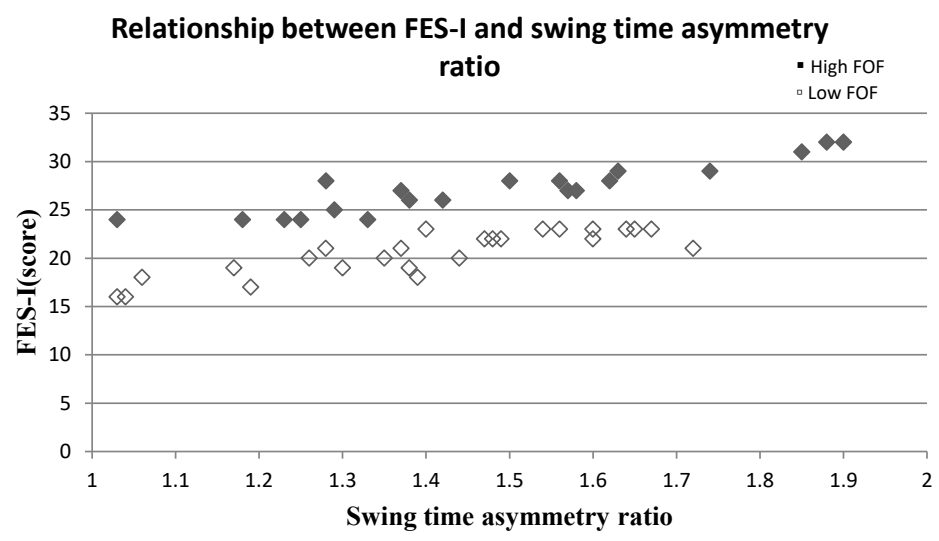

Iranian Rehabilitation Journal

Figure 1. A significant positive relationship was observed between Falls Efficacy Scale-International and swing time asymmetry ratio $(\mathrm{r}=0.65, \mathrm{P}<0.001)(\mathrm{n}=45)$

had a significantly higher AP CoP asymmetry than the individuals without $\mathrm{FoF}(\mathrm{P}<0.033, \mathrm{CI}$ : -0.045 to -0.026$)$. No significant difference was identified in the stance asymmetry and ML CoP asymmetry ratios between the two groups ( $\mathrm{P}<0.821, \mathrm{CI}$ : 0.091 to -0.015 ; $\mathrm{P}<0.801, \mathrm{CI}$ :0.135 to 0.033 , respectively) (Table 2 ).

No significant differences were identified in stance and swing time and step length asymmetry ratios between the participants without FoF and participants with a low FoF (P<0.913, CI: -0.081 to 0.075 ; $\mathrm{P}<0.464$, CI: -0.212 to $0.054 ; \mathrm{P}<0.941, \mathrm{CI}:-0.123$ to 0.067 , respectively). $\mathrm{AP} \mathrm{CoP}$ and ML CoP asymmetry ratios were not significantly different between the two groups $(\mathrm{P}<0.924, \mathrm{CI}$ : -0.036 to 0.026 ; $\mathrm{P}<0.901, \mathrm{CI}:-0.029$ to 0.045 , respectively) (Table 2).

No significant differences were observed in stance and swing time and step length asymmetry ratios between the participants with low and high $\mathrm{FoF}(\mathrm{P}<0.831$, CI: 0.141 to $-0.062 ; \mathrm{P}<0.634$, CI: -0.221 to 0.075 ; $\mathrm{P}<0.085$, CI: -0.008 to 0.183 , respectively). AP CoP and ML CoP asymmetry ratios were not significantly different between the two groups $(\mathrm{P}<0.086, \mathrm{CI}:-0.065$ to 0.003 ; $\mathrm{P}<0.821$, CI: -0.027 to 0.034 , respectively) (Table 2 ).

In individuals with FoF, the level of FoF was related to swing time and step length asymmetry ratios. Increased FoF levels were related to increased swing time asymmetry ( $\mathrm{r}=0.65, \mathrm{P}<0.001, \mathrm{CI}$ : 0.511 to 0.814 ) (Figure 1 ) and step length asymmetry $(\mathrm{r}=0.45, \mathrm{P}<0.002, \mathrm{CI}: 0.166$ to 0.591) (Figure 2). Increased FoF levels were also related to increased AP CoP asymmetry $(\mathrm{r}=0.44, \mathrm{P}<0.003, \mathrm{CI}$ : 0.283 to 0.654 ) (Figure 3 ). No significant association was identified between FES-I scores and the stance asymmetry and ML CoP asymmetry ratios $(\mathrm{r}=0.11, \mathrm{P}<0.452, \mathrm{CI}$ : -0.247 to $0.392 ; \mathrm{r}=0.04, \mathrm{P}<0.716, \mathrm{CI}$ : -0.364 to 0.295 ).

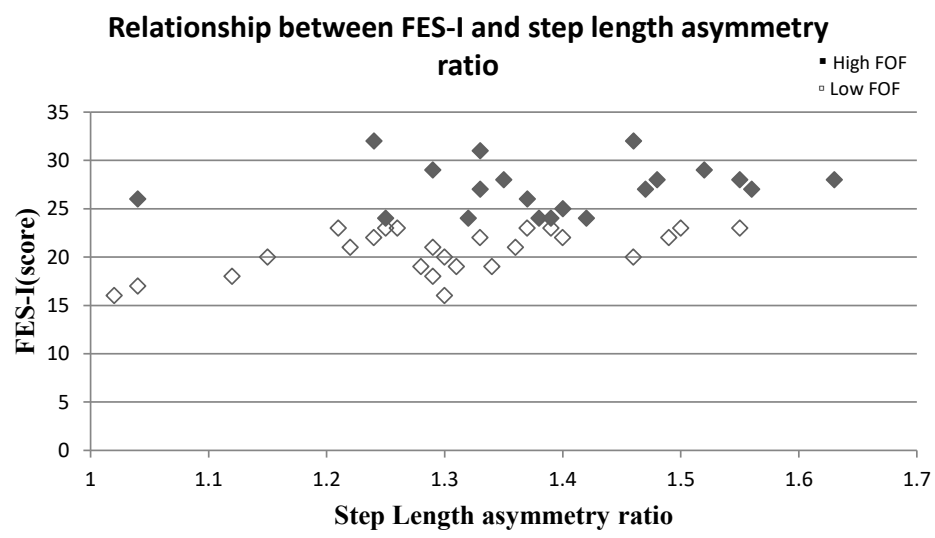

Iranian Rehabilitation Journa

Figure 2. A significant positive relationship was observed between Falls Efficacy Scale-International and step length asymmetry ratio $(\mathrm{r}=0.45, \mathrm{P}<0.002)(\mathrm{n}=45)$. 

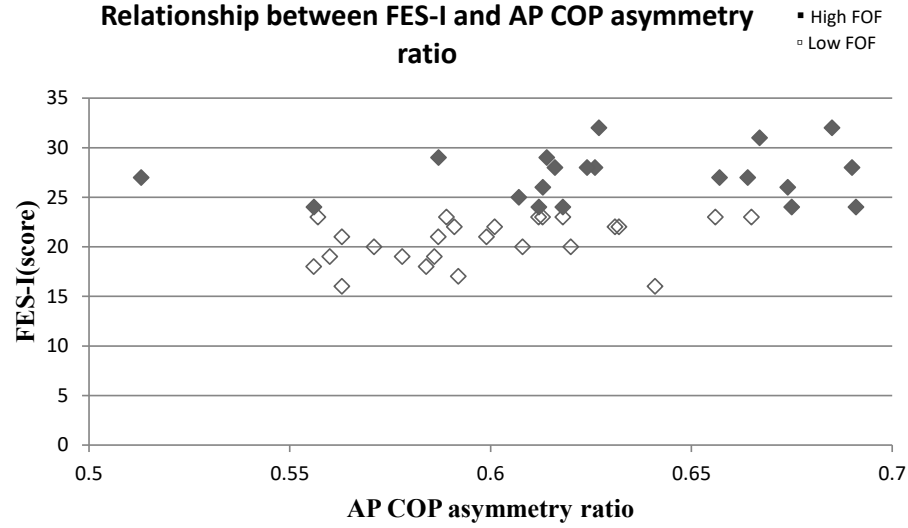

Iranian Rehabilitation Journal

Figure 3. A significant positive relationship was observed between the Falls Efficacy Scale-International and anterior-posterior center of pressure asymmetry ratio $(\mathrm{r}=0.44, \mathrm{P}<0.003)(\mathrm{n}=45)$

\section{Discussion}

Individuals with a high FoF exhibited greater swing time, step length, and AP CoP asymmetry compared to those without FoF. Besides, in participants with FoF, increased FoF levels were related to increased swing time, step length, and AP CoP asymmetry. Contrary to the results of the present study, a study by Schinkel-Ivy et al. in persons with stroke reported no significant difference in gait and balance measures between fear and no fear of falling groups [8]. The differences between the results of these studies are because of the FoF evaluation method [9]. They assessed FoF, using a single question: are you afraid of falling? (Yes or No). Therefore, their study could not determine gait and balance measures in the degree of FoF [9]. However, in the current study, FoF was assessed, using a full questionnaire (FES-I) that evaluates FoF in the context of various physical and social activities [10] and provides more consistency information on the level of FoF [9].

Of the 3 spatiotemporal measures of gait asymmetry, swing time and step length asymmetry were different between the participants without FoF and those with a high FoF. The swing time of the non-paretic leg coincides with the single-limb stance time of the paretic leg, which may provide a significant challenge to the paretic leg. Individuals with a high FoF may decrease the single stance time of the paretic leg to reduce the challenge to it and decrease the risk of the loss of balance during walking, which may lead to a pronounced increase of swing time asymmetry. Step length asymmetry in individuals with a history of stroke is related to the weakness of the affected leg [24].

It is possible that a psychological factor, such as FoF, reinforces these compensatory strategies leading to increased step length asymmetry given that these strate- gies may be adopted to increase stability. In the same vein, shorter stride length and increased stride width were related to FoF in community-dwelling adults [4]. It should be noted that stance time asymmetry was not different among the three groups possibly because two double-support phases of stance time provide sufficient stability during walking.

During standing, AP CoP asymmetry was also greater in participants with a high FoF compared to those without FoF. In other words, the non-paretic leg had a more increased contribution to AP balance control during quiet standing in participants with a high FoF. A previous study by Maki et al. also indicated decreased CoP amplitude in older adults without FoF than those with FoF [25]. They discussed the changes in the muscle tone concerning anxiety, an increase in the voluntary movement, and changes in the concentration of attention as the possible contributing factors to these findings. All of these factors are also considered a possible explanation for the increased contribution of the non-paretic leg to balance control in high FoF individuals with stroke.

Correlational analyses indicated 3 significant associations between the FES-I scores and changes in balance and gait asymmetry ratios. While the causal relationship between FES-I scores and balance and gait asymmetry cannot be determined from the current study, these findings reinforce the influence of FoF in stroke survivors. Hence, there may be a need for treatments to decrease FoF in people after a stroke. It is also useful that therapists assess FoF during the evaluation of balance and gait in individuals with stroke.

There were some limitations to this study. Firstly, the cause and effect relationships could not be assumed because this study was cross-sectional. Secondly, as all 
participants could perform standing and walking trials without assistive devices, the study sample was likely representative of relatively high functioning individuals. This might limit the ability to generalize the current results. Thirdly, it is important to remember that several different physical impairments, including weakness, loss of flexibility, asymmetrical muscular tone, and sensory or cognitive deficits may influence balance and gait asymmetry in individuals with a history of chronic stroke [12, 13]. Unfortunately, we did not measure these potential confounding factors in this study; this issue should be considered in future work.

Despite these limitations, this study was the first research, which provided quantitative evidence establishing balance and gait measures as the important targets for treatment in stroke population relative to the level of FoF. The current study is useful for developing the appropriate interventions in stroke survivors. Based on the current results, it is also useful for clinicians to consider FoF a part of gait and balance evaluations in stroke survivors. Further investigations are also needed to examine whether decreased FoF leads to improvement in gait and balance measures in the stroke population. Most of the participants of this study were also asymmetric in gait and balance measures, suggesting that future work should look at comparing FoF among chronic stroke individuals with symmetric and asymmetric gait and balance. Finally, future studies should consider the effect of potential confounders in the relationship between FoF and gait and balance measures.

\section{Conclusion}

Individuals with stroke with a high FoF exhibited greater gait and balance asymmetry compared to those without FoF. These results suggest that FoF might be considered when planning an intervention for improving balance and gait symmetry.

\section{Ethical Considerations}

\section{Compliance with ethical guidelines}

The research protocol was approved by the Ethical Committee of Mashhad University of Medical Sciences (Code: 89505) and informed consent was provided from all participants.

\section{Funding}

This research was supported by the Vice Chancellor for Research of Mashhad University of Medical Sciences.

\section{Authors' contributions}

Conceptualization, writing-review \& editin and methodology: Mania Sheikh and Hossein Asghar Hosseini; Investigation: Mania Sheikh; Funding acquisition, resources, and supervision: Hossein Asghar Hosseini.

\section{Conflict of interest}

The authors declared no conflict of interest.

\section{Acknowledgments}

The authors would like to thank the clinicians, who helped with recruiting participants for the study and the participants for their cooperation.

\section{References}

[1] Weerdesteyn V, de Niet M, van Duijnhoven HJR, Geurts $\mathrm{ACH}$. Falls in individuals with stroke. The Journal of Rehabilitation Research and Development. 2008; 45(8):1195-213. [DOI:10.1682/JRRD.2007.09.0145] [PMID]

[2] Batchelor F, Hill K, Mackintosh Sh, Said C. What works in falls prevention after stroke? A systematic review and meta-analysis. Stroke. 2010; 41(8):1715-22. [DOI:10.1161/ STROKEAHA.109.570390] [PMID]

[3] Batchelor FA, Mackintosh SF, Said CM, Hill KD. Falls after stroke. International Journal of Stroke. 2012; 7(6):482-90. [DOI:10.1111/j.1747-4949.2012.00796.x] [PMID]

[4] Chamberlin ME, Fulwider BD, Sanders SL, Medeiros JM Does fear of falling influence spatial and temporal gait parameters in elderly persons beyond changes associated with normal aging? The Journals of Gerontology: Series A. 2005; 60(9):1163-7. [DOI:10.1093/gerona/60.9.1163] [PMID]

[5] Makino K, Makizako H, Doi T, Tsutsumimoto K, Hotta R, Nakakubo Sh, et al. Impact of fear of falling and fall history on disability incidence among older adults: Prospective cohort study. International Journal of Geriatric Psychiatry. 2018; 33(4):658-62. [DOI:10.1002/gps.4837] [PMID]

[6] Makino K, Makizako H, Doi T, Tsutsumimoto K, Hotta R, Nakakubo $S$, et al. Fear of falling and gait parameters in older adults with and without fall history. Geriatrics \& Gerontology International. 2017; 17(12):2455-9. [DOI:10.1111/ggi.13102] [PMID]

[7] Allali G, Ayers EI, Holtzer R, Verghese J. The role of postural instability/gait difficulty and fear of falling in predicting falls in non-demented older adults. Archives of Gerontology and Geriatrics. 2017; 69:15-20. [DOI:10.1016/j.archger.2016.09.008] [PMID] [PMCID]

[8] Schinkel-Ivy A, Inness EL, Mansfield A. Relationships between fear of falling, balance confidence, and control of balance, gait, and reactive stepping in individuals with subacute stroke. Gait \& Posture. 2016; 43:154-9. [DOI:10.1016/j. gaitpost.2015.09.015] [PMID] [PMCID] 
[9] Ayoubi F, Launay CP, Annweiler C, Beauchet O. Fear of falling and gait variability in older adults: A systematic review and meta-analysis. Journal of the American Medical Directors Association. 2015; 16(1):14-9. [DOI:10.1016/j.jamda.2014.06.020] [PMID]

[10] Delbaere K, Close JC, Mikolaizak AS, Sachdev PS, Brodaty H, Lord SR. The Falls Efficacy Scale International (FES-I). A comprehensive longitudinal validation study. Age and Ageing. 2010; 39(2):210-6. [DOI:10.1093/ageing/afp225] [PMID]

[11] Patterson KK, Parafianowicz I, Danells CJ, Closson V, Verrier MC, Staines WR, et al. Gait asymmetry in communityambulating stroke survivors. Archives of Physical Medicine and Rehabilitation. 2008; 89(2):304-10. [DOI:10.1016/j. apmr.2007.08.142] [PMID]

[12] Hsu AL, Tang PF, Jan MH. Analysis of impairments influencing gait velocity and asymmetry of hemiplegic patients after mild to moderate stroke. Archives of Physical Medicine and Rehabilitation. 2003; 84(8):1185-93. [DOI:10.1016/S00039993(03)00030-3]

[13] Genthon N, Rougier P, Gissot AS, Froger J, Pelissier J, Perennou D. Contribution of each lower limb to upright standing in stroke patients. Stroke. 2008; 39(6):1793-9. [DOI:10.1161/ STROKEAHA.107.497701] [PMID]

[14] Goldstein M, Barnett HJM, Orgogozo JM, Sartorius N, Symon L, Vereshchagin NV. Stroke--1989. Recommendations on stroke prevention, diagnosis, and therapy. Report of the WHO task force on Stroke and other cerebrovascular disorders. Stroke. 1989; 20(10):1407-31. [DOI:10.1161/01. STR.20.10.1407] [PMID]

[15] Tuijl JP, Scholte EM, de Craen AJM, van der Mast RC. Screening for cognitive impairment in older general hospital patients: Comparison of the six-item cognitive impairment test with the mini-mental state examination. International Journal of Geriatric Psychiatry. 2012; 27(7):755-62. [DOI:10.1002/ gps.2776] [PMID]

[16] Ansari NN, Naghdi S, Arab TK, Jalaie S. The interrater and intrarater reliability of the Modified Ashworth Scale in the assessment of muscle spasticity: Limb and muscle group effect. NeuroRehabilitation. 2008; 23(3):231-7. [DOI:10.3233/NRE2008-23304] [PMID]

[17] Gladstone DJ, Danells CJ, Black SE. The fugl-meyer assessment of motor recovery after stroke: A critical review of its measurement properties. Neurorehabilitation and Neural Repair 2002; 16(3):232-40. [DOI:10.1177/154596802401105171] [PMID]

[18] Azad A, Hassani Mehraban A, Mehrpour M, Mohammadi B. Clinical assessment of fear of falling after stroke: Validity, reliability and responsiveness of the Persian version of the Fall Efficacy Scale-International. Medical Journal of the Islamic Republic of Iran. 2014; 28:131. [PMID]

[19] Mansfield A, Danells CJ, Inness E, Mochizuki G, McIlroy WE. Between-limb synchronization for control of standing balance in individuals with stroke. Clinical Biomechanics (Bristol, Avon). 2011; 26(3):312-7. [DOI:10.1016/j.clinbiomech.2010.10.001] [PMID]

[20] Hendrickson J, Patterson KK, Inness EL, Mcllroy WE, Mansfield A. Relationship between asymmetry of quiet standing balance control and walking post-stroke. Gait \& Posture. 2014; 39(1):177-81. [DOI:10.1016/j.gaitpost.2013.06.022] [PMID]
[21] Soda P, Carta A, Formica D, Guglielmelli E. A low-cost video-based tool for clinical gait analysis. Paper presented at: Annual International Conference of the IEEE Engineering in Medicine and Biology Society. 3-6 September 2009; Minneapolis, MN, USA. [DOI:10.1109/IEMBS.2009.5333623] [PMID]

[22] Churchill AJG, Halligan PW, Wade DT. RIVCAM: A simple video-based kinematic analysis for clinical disorders of gait. Computer Methods and Programs in Biomedicine. 2002; 69(3):197-209. [DOI:10.1016/S0169-2607(01)00191-2]

[23] Patterson KK, Gage WH, Brooks D, Black SE, Mcllroy WE. Evaluation of gait symmetry after stroke: A comparison of current methods and recommendations for standardization. Gait \& Posture. 2010; 31(2):241-6. [DOI:10.1016/j.gaitpost.2009.10.014] [PMID]

[24] Balasubramanian CK, Bowden MG, Neptune RR, Kautz SA. Relationship between step length asymmetry and walking performance in subjects with chronic hemiparesis. Archives of Physical Medicine and Rehabilitation. 2007; 88(1):439. [DOI:10.1016/j.apmr.2006.10.004] [PMID]

[25] Maki BE, Holliday PJ, Topper AK. Fear of falling and postural performance in the elderly. Journal of Gerontology. 1991; 46(4):M123-M131. [DOI:10.1093/geronj/46.4.M123] [PMID] 